\title{
The top quark found at long last
}

The announcement from Fermilab last week is neither a mere formality nor the capstone of a theoretical edifice, but another landmark in the long road to telling how matter is constructed.

THE announcement at Fermilab that two detectors catching decay products from particles formed in proton-antiproton collisions have finally provided clinching evidence that the 'top' quark exists has an unavoidable sense of anticlimax. People have been talking about it for a long time, after all. And given the way in which the quark explanation of nuclear matter has proved over the years to have predictive power, what more natural than that its existence should be taken for granted?

But that would be mistaken. Science hangs on the empirical discovery of what the real world is like. However solid the theoretical basis of some field of inquiry may be, and however confident people may be about its predictions, experimental verification does more than confirm people in their prejudices. Often, verification is a dramatic reminder of the poverty of human imagination.

So much is clear from the history of the idea of neutron stars. The idea that a sufficiently massive star that had exhausted its thermonuclear fuel might collapse into a mass of neutrons appears first to have been suggested by Baade and Zwicky in 1935. Their natural conclusion was that such an object would be relatively dull, a massive object emitting no energy worth talking of and thus virtually undetectable. But real-life neutron stars, when discovered in 1969 as pulsating stars, have proved to be among the most spectacular and interesting objects in the Galaxy. Who predicted that that would be the case?

The top quark (called ' $t$ ') is potentially a much simpler entity. The theorists, building on Murray Gell-Mann's original idea, say that there should be six quarks, with the whimsical names of 'up', 'down', 'strange', 'charm', 'bottom' and 'top', or u, $\mathrm{d}, \mathrm{s}, \mathrm{c}, \mathrm{b}$ and $\mathrm{t}$. Their function is to constitute the more familiar particles of nuclear matter. A proton consists of two u's and one $d$, for example, a $\pi^{+}$meson of one $u$ and an anti-d.

Because, like electrons, quarks have half-integral spin, each quark has an antiparticle doppelgänger, with identical mass, but opposite electric charge. There is now even more than a hint that the number of quark 'generations' (the pairs $\mathrm{u}$ and $\mathrm{d}, \mathrm{s}$ and $\mathrm{c}, \mathrm{b}$ and t) must be limited to three, but that hangs on the assumption that the three known forms of neutrino have no mass, which may be false. Otherwise, the discovery of $t$ seems neatly to fill out the list of the expected quarks. Why should not physics now turn to simpler things?

First, there is the business of the mass of the top quark reported last week. One group, that responsible for the D0 detector, reports a mass equivalent to $199 \mathrm{GeV}$; the other, a mass equivalent to $176 \mathrm{GeV}$. (The two estimates are consistent with each other, given that the first group quotes statistical and systematic errors of \pm 20 and \pm 22 respectively, and the second group, \pm 8 and \pm 10 .) These values are much greater than expected only a few years ago, implying that each $t$ is some 200,000 times more massive than a proton, and more than 10 million times as massive as a $\mathrm{u}$. In themselves, they explain why the top quark has been so long waiting in the wings. The beams of protons and antiprotons from the Tevatron at Fermilab have been generating particles with an energy equivalent to $1.8 \mathrm{TeV}$ (10 per cent below the maximum design energy) for months now, and there are still only a few handfuls of significant events to boast about.

All the other quarks are much less massive. The lightest is $\mathrm{u}$, with a mass of a few $\mathrm{MeV}$, next comes $\mathrm{d}$, between 1.5 and 4 times as massive, then s (more than 100 $\mathrm{MeV}$ ), c (about $1.5 \mathrm{GeV}$ ) and b (about 5 $\mathrm{GeV}$ ). Why should t be up to twenty times more massive than its partner? Nobody seems very clear. Nor is there any very clear explanation of the 25 -fold mass difference between s and its partner, $\mathrm{c}$.

Indeed, there is bound to be ambiguity about what exactly is meant by the mass of a single quark, when quarks seem not to exist except in combinations with others. The Fermilab measurements are essentially measurements of the mass of the meson-like entity $\bar{t}$, the combination of top and antitop.

The implicit assumption is that the mass of each quark is half that of the combination. But quarks are supposed to be held together by gluons, while a certain amount of binding energy must be involved. Half the mass of the $\bar{t}$ pair may bear very little relation to the mass of a bare quark, or even to the values that the theoreticians should enter into the equations they have lovingly constructed. The hope is that all this will become plain when the Higgs particle is found.

Other issues are even more perplexing, and not least what is called the 'confinement' problem: why do not bare quarks exist? It may, of course, be relevant that quarks have electrical charges that are not integral multiples of the unit electronic charge $(\mathrm{d}, \mathrm{s}$ and $\mathrm{b}$ have charge $-1 / 3, \mathrm{u}$, $c$ and $t$ have charge $+2 / 3$ ), and that the space-time in which we live will not accommodate non-integral charges, but that is simply a way of replacing one conundrum by another. "Why not?" is the natural and proper response.

Another aspect of that problem, the less serious restrictions there appear to be on the occurrence of quark composites, has however been accommodated by the invention of a novel qualitative property of all quarks: colour. Colour is a kind of analogue of electric charge, except that it comes in three forms ('red', 'green' and 'blue', of course). Like colours repel, unlike colours attract, so a composite of two quarks will hold together only if they are differently coloured, whereupon only a quark of the third colour can be added in a stable fashion to the composite.

The notion of colour is the source of the prefix in 'quantum chromodynamics', the field theory of nuclear matter. It is the analogue of the successful theory of quantum electrodynamics in this more difficult field. By common agreement, it is an awkward theory to calculate with, yet that has not prevented people from using it as a component in the formulation of the grand unified theories, called 'GUTs', which are sometimes expected to amount to the famous 'theory of everything'.

It is easy to make fun of this enterprise, but it is also unfair. We forget that GellMann's first version of the quark model (when three rather than six quarks seemed sufficient) seemed in the late 1950 s to be an over-imaginative and even artificial way of categorizing the particles then known. It became respectable after the prediction (by Gell-Mann in 1962) of a particle called $\Omega^{-}$was verified. (The $\Omega^{-}$ is a composite of three $s$ quarks which, because of their identity, cannot be in the same state and so must have spin $3 \times 1 / 2$.)

Who can say that the same will not happen with the coloured-quark view now in vogue? The only certainty is that much more will have to be learned in the laboratory of how quarks behave before there is grist for the mill of speculation and prediction. And even then, there will be much deeper questions about the link between what nature allows and the structure of space-time crying out for attention. The discovery of the top quark is just another landmark in the long process of learning how matter is constructed. John Maddox 